\title{
On the Connecting Thickness of Arithmetical Discrete Planes
}

\author{
Eric Domenjoud ${ }^{1}$, Damien Jamet ${ }^{1}$, and Jean-Luc Toutant ${ }^{2}$ \\ 1 Loria - Université Nancy 1 - CNRS, Campus Scientifique, BP 239, 54506 \\ Vandœuvre-lès-Nancy, France \\ eric.domenjoud@loria.fr, damien.jamet@loria.fr \\ 2 LAIC, IUT Clermont-Ferrand, Campus des Cézeaux, 63172 Aubière Cedex \\ toutant@iut.u-clermont1.fr
}

\begin{abstract}
While connected rational arithmetical discrete lines and connected rational arithmetical discrete planes are entirely characterized, only partial results exist for the irrational arithmetical discrete planes. In the present paper, we focus on the connectedness of irrational arithmetical discrete planes, namely the arithmetical discrete planes with a normal vector of which the coordinates are not $\mathbb{Q}$-linear dependent. Given $\mathbf{v} \in \mathbb{R}^{3}$, we compute the lower bound of the thicknesses 2-connecting the arithmetical discrete planes with normal vector $\mathbf{v}$. In particular, we show how the translation parameter operates in the connectedness of the arithmetical discrete planes.
\end{abstract}

\section{Introduction}

In [1], J.-P. Reveillès introduced arithmetical discrete lines as sets of pairs of integers satisfying a double Diophantine inequality. The arithmetical discrete line with normal vector $\mathbf{v} \in \mathbb{R}^{2} \backslash\{(0,0)\}$, translation parameter $\mu \in \mathbb{R}$ and arithmetical thickness $\omega \in \mathbb{R}$ is the set $\mathbb{L}(\mathbf{v}, \mu, \omega)$ defined by:

$$
\mathbb{L}(\mathbf{v}, \mu, \omega)=\left\{\mathbf{x}=\left(x_{1}, x_{2}\right) \in \mathbb{Z}^{2} ; 0 \leq\langle\mathbf{v}, \mathbf{x}\rangle+\mu<\omega\right\},
$$

where $\langle\mathbf{v}, \mathbf{x}\rangle=v_{1} x_{1}+v_{2} x_{2}$.

The definition of arithmetical discrete lines extends naturally to the definition of arithmetical discrete planes in the 3 -dimensional discrete space $\mathbb{Z}^{3}$, and to the definition of arithmetical discrete hyperplanes in higher dimensions [2].

The problem of computing the minimal thickness connecting an arithmetical discrete plane has already been treated in several works [3456]. While all these past works deal with rational arithmetical discrete planes (or hyperplanes), that is, with a normal vector $\mathbf{v}$ which can be expressed as an integer vector, the present work deals with any arithmetical discrete plane. More precisely, we deal with the following questions: given $\mathbf{v}=\left(v_{1}, v_{2}, v_{3}\right) \in$ $\mathbb{R}^{3} \backslash\left\{(0,0,0\}\right.$ with $\operatorname{dim}_{\mathbb{Q}}\left\{v_{1}, v_{2}, v_{3}\right\} \neq 1$, given $\mu \in \mathbb{R}$, how much is $\inf \{\omega \in$ $\mathbb{R},\left\{\mathbf{x}=\left(x_{1}, x_{2}, x_{3}\right) \in \mathbb{Z}^{3} ; 0 \leq v_{1} x_{1}+v_{2} x_{2}+v_{3} x_{3}+\mu<\omega\right\}$ is connected $\}$ ?

The present paper is organized as follows. After having introduced the basic notions and notations we use throughout the present paper in 
Section 2 2 we deal with the connectedness of arithmetical discrete lines in Section 13. In Section 4, we deal with the connectedness of arithmetical discrete planes and show how to reduce the computation of $\Omega(\mathbf{v}, \mu)=\inf \{\omega \in$ $\mathbb{R},\left\{\mathbf{x}=\left(x_{1}, x_{2}, x_{3}\right) \in \mathbb{Z}^{3} ; 0 \leq v_{1} x_{1}+v_{2} x_{2}+v_{3} x_{3}+\mu<\omega\right\}$ is connected $\}$ to the one of a smaller vector (see Theorem 3 and Corollary 2). In Section 5, we are interested in computing explicitly $\Omega(\mathbf{v}, \mu)$ and we provide formulas for two special cases.

\section{Basic Notions and Notation}

The aim of this section is to introduce the basic notions and notation we use throughout the present paper.

Let $n$ be an integer equal or greater than 2 . Let $\left\{\mathbf{e}_{\mathbf{1}}, \mathbf{e}_{\mathbf{2}}, \ldots, \mathbf{e}_{\mathbf{n}}\right\}$ denote the canonical basis of the Euclidean vector space $\mathbb{R}^{3}$. Let us call discrete set, any subset of the discrete space $\mathbb{Z}^{n}$. In the following, for the sake of clarity, we denote by $\left(x_{1}, x_{2}, \ldots, x_{n}\right)$ the point $\mathbf{x}=\sum_{i=1}^{n} x_{i} \mathbf{e}_{\mathbf{i}} \in \mathbb{R}^{n}$. An integer point $\mathbf{x} \in \mathbb{Z}^{n}$ is called a voxel.

Definition 1 ( $\kappa$-Adjacency). Let $\kappa \in\{0,1, \ldots, n-1\}$. Two voxels $\mathbf{x}, \mathbf{y} \in \mathbb{Z}^{n}$ are $\kappa$-adjacent if:

$$
\|\mathbf{x}-\mathbf{y}\|_{\infty}=1 \text { and }\|\mathbf{x}-\mathbf{y}\|_{1} \leq n-\kappa
$$

REMARK - $\|\mathbf{x}\|_{\infty}=\max _{i \in\{1,2, \ldots, n\}}\left\{\left|x_{i}\right|\right\}$ and $\|\mathbf{x}\|_{1}=\sum_{i=1}^{n}\left|x_{i}\right|$.

In other words, the voxel $\mathbf{x}$ and the voxel $\mathbf{y}$ are $\kappa$-adjacent if they are distinct, if the differences of their coordinates are at most 1 and $\mathbf{x}$ and $\mathbf{y}$ have at most $n-\kappa$ different coordinates. A $\kappa$-path is a (finite or infinite) sequence of consecutive $\kappa$-adjacent voxels. If $\left(\gamma_{i}\right)_{1 \leq i \leq k}$ is a finite $\kappa$-path, then we say that $\gamma$ links the voxel $\gamma_{1}$ to the voxel $\gamma_{k}$.

Definition 2 ( $\kappa$-Connected Sets). Let $E$ be a discrete set and let $\kappa \in$ $\{0,1, \ldots, n-1\}$. Then $E$ is $\kappa$-connected if, for each pair of voxels $(\mathbf{x}, \mathbf{y}) \in E^{2}$, there exists a $\kappa$-path in E linking $\mathbf{x}$ to $\mathbf{y}$.

In [1, J.-P. Reveillès introduced the arithmetical discrete line as a set of integer points satisfying a double Diophantine inequality.

Definition 3 (Arithmetical Discrete Lines [1]). Let $\mathbf{v} \in \mathbb{Z}^{2} \backslash\{(0,0)\}, \mu \in \mathbb{Z}$ and $\omega \in \mathbb{Z}$ with $\operatorname{gcd}\left\{v_{1}, v_{2}\right\}=1$. The arithmetical discrete line $\mathbb{L}(\mathbf{v}, \mu, \omega)$ with normal vector $\mathbf{v}$, translation parameter $\mu$ and arithmetical thickness $\omega$ is the discrete set defined by:

$$
\mathbb{L}(\mathbf{v}, \mu, \omega)=\left\{\mathbf{x} \in \mathbb{Z}^{2} ; 0 \leq\langle\mathbf{v}, \mathbf{x}\rangle+\mu<\omega\right\},
$$

where $\langle\mathbf{v}, \mathbf{x}\rangle=v_{1} x_{1}+v_{2} x_{2}$. 
Let us notice that Definition 3 deals only with integer parameters $\mathbf{v}, \mu$ and $\omega$. In fact, this definition extends in a natural way to any parameters (integer or not) and in higher dimensions as follows:

Definition 4 (Arithmetical Discrete Hyperplanes [1,2]). Let $n \in \mathbb{N}$ greater than 2 , let $\mathbf{v} \in \mathbb{R}^{n} \backslash\{(0, \ldots, 0)\}, \mu \in \mathbb{R}$ and $\omega \in \mathbb{R}$. The arithmetical discrete hyperplane $\mathbb{H}(\mathbf{v}, \mu, \omega)$ with normal vector $\mathbf{v}$, translation parameter $\mu$ and arithmetical thickness $\omega$ is the discrete set defined by:

$$
\mathbb{H}(\mathbf{x}, \mu, \omega)=\left\{\mathbf{v} \in \mathbb{Z}^{n} ; 0 \leq\langle\mathbf{v}, \mathbf{x}\rangle+\mu<\omega\right\}
$$

where $\langle\mathbf{v}, \mathbf{x}\rangle=v_{1} x_{1}+\cdots+v_{n} x_{n}$.

If $n=3$, the arithmetical discrete hyperplane $\mathbb{H}(\mathbf{v}, \mu, \omega)$ is called an arithmetical discrete plane and is denoted $\mathbb{P}(\mathbf{v}, \mu, \omega)$.

Let us first recall a useful lemma concerning a particular subclass of the arithmetical discrete planes:

Lemma 1 ([7]). Let $n \in \mathbb{N}$ greater than 2 , let $\mathbf{v} \in \mathbb{R}^{n} \backslash\{(0, \ldots, 0)\}, \mu \in \mathbb{R}$ and $\omega \in \mathbb{R}$.

i) If $\operatorname{dim}_{\mathbb{Q}}\left\{v_{1}, \ldots, v_{n}\right\}=1$, then there exist $\mathbf{v}^{\prime} \in \mathbb{Z}^{n}, \mu^{\prime} \in \mathbb{Z}$ and $\omega^{\prime} \in \mathbb{Z}$ with $\operatorname{gcd}\left\{v_{1}^{\prime}, \ldots, v_{n}^{\prime}\right\}=1$ such that $\mathbb{H}(\mathbf{v}, \mu, \omega)=\mathbb{H}\left(\mathbf{v}^{\prime}, \mu^{\prime}, \omega^{\prime}\right)$.

ii) Let $\mathbf{v}^{\prime} \in \mathbb{R}^{n} \backslash\{(0, \ldots, 0)\}, \mu^{\prime} \in \mathbb{R}$ and $\omega^{\prime} \in \mathbb{R}$. If $\mathbb{H}(\mathbf{v}, \mu, \omega)=\mathbb{H}\left(\mathbf{v}^{\prime}, \mu^{\prime}, \omega^{\prime}\right)$ then the vectors $\mathbf{v}$ and $\mathbf{v}^{\prime}$ are colinear.

Definition 5 (Rational Arithmetical Discrete Hyperplanes). Let $n \in \mathbb{N}$ greater than 2 , let $\mathbf{v} \in \mathbb{R}^{n} \backslash\{(0, \ldots, 0)\}, \mu \in \mathbb{R}$ and $\omega \in \mathbb{R}$. If $\operatorname{dim}_{\mathbb{Q}}\left\{v_{1}, \ldots, v_{n}\right\}=$ 1 , then the arithmetical discrete hyperplane $\mathbb{H}(\mathbf{v}, \mu, \omega)$ and its normal vector $\mathbf{v}$ are said to be rational.

REMARK - Throughout the present paper, if $\mathbb{P}(\mathbf{v}, \mu, \omega)$ is a rational arithmetical plane, then we assume, with no loss of generality, $\mathbf{v} \in \mathbb{Z}^{3}$ and $\mu \in \mathbb{Z}$.

\section{1-Connectedness of (Rational or Irrational) Arithmetical Discrete Lines}

Definition 6 (Minimal 1-Connecting Thickness). Let $\mathbf{v} \in \mathbb{R}^{2} \backslash\{(0,0)\}$ and $\mu \in \mathbb{R}$. The minimal 1-connecting thickness of $(\mathbf{v}, \mu)$ is the number $\Omega_{1}(\mathbf{v}, \mu)$ defined by:

$$
\Omega_{1}(\mathbf{v}, \mu)=\inf \{\omega \in \mathbb{R} ; \mathbb{L}(\mathbf{v}, \mu, \omega) \text { is 1-connected }\} .
$$

In [1], J.-P. Reveillès showed how the 1-connectedness of a rational arithmetical discrete line is entirely determined by its normal vector and its thickness. More precisely:

Theorem 1 ([1]). Let $\mathbb{L}(\mathbf{v}, \mu, \omega)$ be a rational arithmetical discrete line with $\omega \in \mathbb{Z}$ and $\operatorname{gcd}\left\{v_{1}, v_{2}\right\}=1$. The arithmetical discrete line $\mathbb{L}(\mathbf{v}, \mu, \omega)$ is 1-connected if and only if $\omega \geq\|\mathbf{v}\|_{1}$. 
Theorem 3 holds only for integer normal vectors with coprime coordinates. Moreover, if one does not suppose $\operatorname{gcd}\left\{v_{1}, v_{2}\right\}=1$, then Theorem 3 does not hold any more. For instance let us consider $\mathbf{v}=0 \mathbf{e}_{\mathbf{1}}+v_{2} \mathbf{e}_{\mathbf{2}}$. For all $\mu \in \mathbb{R}$ and $\omega \in \mathbb{R}$, one checks:

$$
\mathbb{L}(\mathbf{v}, \mu, \omega) \neq \emptyset \Longleftrightarrow \mathbb{L}(\mathbf{v}, \mu, \omega) \text { is } 1 \text {-connected } \Longleftrightarrow \omega \geq \mu-v_{2}\left\lfloor\frac{\mu}{v_{2}}\right\rfloor .
$$

A direct extension of Theorem 3 to any rational arithmetical discrete lines becomes:

Corollary 1. Let $\mathbf{v} \in \mathbb{Z}^{2} \backslash\{(0,0)\}$ and $\mu \in \mathbb{Z}$.

$$
\begin{array}{ll}
\Omega_{1}(\mathbf{v}, \mu)=\mu-v_{2}\left\lfloor\frac{\mu}{v_{2}}\right\rfloor, & \text { if } v_{1}=0 \text { and } v_{2} \neq 0, \\
\Omega_{1}(\mathbf{v}, \mu)=\mu-v_{1}\left\lfloor\frac{\mu}{v_{1}}\right\rfloor, & \text { if } v_{1} \neq 0 \text { and } v_{2}=0, \\
\Omega_{1}(\mathbf{v}, \mu)=\|\mathbf{v}\|_{1}-\operatorname{gcd}\left\{v_{1}, v_{2}\right\}, & \text { if } v_{1} \neq 0 \text { and } v_{2} \neq 0 .
\end{array}
$$

REMARK - The third item of Corollary is not in contradiction with Theorem. Indeed, let $\mathbf{v} \in \mathbb{Z}^{2} \backslash\{(0,0)\}, \mu \in \mathbb{Z}$ and $\left(x_{1}, x_{2}\right) \in \mathbb{Z}^{2}$, then one has:

$$
v_{1} x_{1}+v_{2} x_{2}+\mu<\|\mathbf{v}\|_{1} \Longleftrightarrow v_{1} x_{1}+v_{2} x_{2}+\mu \leq\|\mathbf{v}\|_{1}-\operatorname{gcd}\left\{v_{1} v_{2}\right\} .
$$

Let us now extend Theorem 3 and Corollary 3 to irrational arithmetical discrete lines. One of our main ambition is to exhibit a unique formulation the properties we state. For that purpose, we introduce the following notation.

Notation — Let $\Phi: \mathbb{R}^{2} \longmapsto \mathbb{R}^{2}$ the map defined by:

$$
\begin{aligned}
\Phi: \mathbb{R}^{2} & \longmapsto \mathbb{R}^{2} \\
(x, y) & \mapsto \begin{cases}\left(y-\left\lfloor\frac{y}{x}\right\rfloor x, x\right), & \text { if } x \neq 0, \\
(x, y) & \text { otherwise. }\end{cases}
\end{aligned}
$$

Given $x \in \mathbb{R}$ and $y \in \mathbb{R}$, we set $x \wedge y=\lim _{n \rightarrow \infty}\left|\left(\Phi^{n}(x, y)\right)_{2}\right|$.

It is clear that, given $(x, y) \in \mathbb{Z}^{2}, x \wedge y=\operatorname{gcd}\{x, y\}$. Moreover, one states:

Lemma 2. Let $(x, y, z) \in \mathbb{R}^{3}$ and $\lambda \in \mathbb{R}$.

1. $\lambda x \wedge \lambda y=|\lambda|(x \wedge y)$.

2. if $\operatorname{dim}_{\mathbb{Q}}\{x, y\}=2$, then $x \wedge y=0$.

3. $x \wedge(y \wedge z)=(x \wedge y) \wedge z$.

Notation - Thanks to Lemma 2, for any $x_{1}, x_{2}, \ldots, x_{k} \in \mathbb{R}$, we denote $x_{1} \wedge$

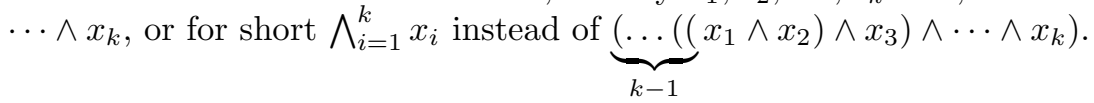

We now state the main result of the present section: 
Theorem 2. Let $\mathbf{v} \in \mathbb{R}^{2} \backslash\{(0,0)\}$ and $\mu \in \mathbb{R}$. Then,

$$
\begin{array}{ll}
\Omega_{1}(\mathbf{v}, \mu)=\mu-v_{2}\left\lfloor\frac{\mu}{v_{2}}\right\rfloor, & \text { if } v_{1}=0 \text { and } v_{2} \neq 0, \\
\Omega_{1}(\mathbf{v}, \mu)=\mu-v_{1}\left\lfloor\frac{\mu}{v_{1}}\right\rfloor, & \text { if } v_{1} \neq 0 \text { and } v_{2}=0, \\
\Omega_{1}(\mathbf{v}, \mu)=\|\mathbf{v}\|_{1}-v_{1} \wedge v_{2}, & \text { if } v_{1} \neq 0 \text { and } v_{2} \neq 0 .
\end{array}
$$

Proof (Sketch). The case $\operatorname{dim}_{\mathbb{Q}}\left\{v_{1}, v_{2}\right\}=1$ (in particular if $v_{1}=0$ or $v_{2}=$ 0) has already been treated (see Corollary 3 and Lemma 1). Assume that $\operatorname{dim}_{\mathbb{Q}}\left\{v_{1}, v_{2}\right\}=2$. Then $v_{1} \wedge v_{2}=0$. With no loss of generality, assume $0 \leq v_{1} \leq v_{2}$ (one reduces to this assumption, up to an isomorphism of the unit cube $\left.\left[-\frac{1}{2}, \frac{1}{2}\right]^{3}\right)$.

i) The projection $\pi: \mathbb{L}\left(\mathbf{v}, \mu,\|\mathbf{v}\|_{1}\right) \longrightarrow \mathbb{Z}$ along the vector $\mathbf{e}_{\mathbf{1}}+\mathbf{e}_{\mathbf{2}}$ is one-toone. Given $\mathbf{x}$ and $\mathbf{y}$ in $\mathbb{L}\left(\mathbf{v}, \mu,\|\mathbf{v}\|_{1}\right)$, one shows that $\mathbf{x}$ and $\mathbf{y}$ are 1-adjacent if, and only if, $|\pi(\mathbf{x})-\pi(\mathbf{y})|=1$. We deduce that the arithmetical discrete line $\mathbb{L}\left(\mathbf{v}, \mu,\|\mathbf{v}\|_{1}\right)$ is 1 -connected.

ii) Let $\omega \geq\|\mathbf{v}\|_{1}$ and let $\mathbf{x} \in \mathbb{L}(\mathbf{v}, \mu, \omega)$. Then $\mathbf{x}$ is either in $\mathbb{L}\left(\mathbf{v}, \mu,\|\mathbf{v}\|_{1}\right)$ or vertically 1 -linked with an element of $\mathbb{L}\left(\mathbf{v}, \mu,\|\mathbf{v}\|_{1}\right)$. Hence $\mathbb{L}(\mathbf{v}, \mu, \omega)$ is 1-connected.

iii) Let $\omega \in\left[0,\|\mathbf{v}\|_{1}\left[\right.\right.$. Since $\operatorname{dim}_{\mathbb{Q}}\left\{v_{1}, v_{2}\right\}=2$, the set $\{\langle\mathbf{v}, \mathbf{x}\rangle+\mu, \mathbf{x} \in$ $\left.\mathbb{L}\left(\mathbf{v}, \mu,\|\mathbf{v}\|_{1}\right)\right\}$ is dense in $\left[0,\|\mathbf{v}\|_{1}\left[\right.\right.$. Let $\mathbf{x} \in \mathbb{L}\left(\mathbf{v}, \mu,\|\mathbf{v}\|_{1}\right)$ such that $\langle\mathbf{v}, \mathbf{x}\rangle+\mu \in\left[\omega,\|\mathbf{v}\|_{1}\left[\right.\right.$. Then, $\mathbb{L}(\mathbf{v}, \mu, \omega) \subseteq \mathbb{L}\left(\mathbf{v}, \mu,\|\mathbf{v}\|_{1}\right) \backslash\{x\}$. From $\left.\mathrm{i}\right)$, we state that the set $\mathbb{L}\left(\mathbf{v}, \mu,\|\mathbf{v}\|_{1}\right) \backslash\{x\}$ is not 1 -connected, and so is $\mathbb{L}(\mathbf{v}, \mu, \omega)$.

\section{2-Connectedness of Arithmetical Discrete Planes}

The case of arithmetical discrete lines is somewhat confusing. Indeed, while an arithmetical discrete line is 1-connected if, and only if, it separates the discrete space $\mathbb{Z}^{2}$ into two 0-connected components, there exist 2-connected arithmetical discrete planes $\mathbb{P}(\mathbf{v}, \mu, \omega)$ with $\omega<\|\mathbf{v}\|_{1}$, that is, which do not separate the discrete space $\mathbb{Z}^{3}$ into two 0-connected components [2].

Definition 7 (Minimal 2-Connecting Thickness). Let $\mathbf{v} \in \mathbb{R}^{3} \backslash\{(0,0,0\}$ and $\mu \in \mathbb{R}$. The minimal 2-connecting thickness of $(\mathbf{v}, \mu)$ is the number $\Omega_{2}(\mathbf{v}, \mu)$ defined by:

$$
\Omega_{2}(\mathbf{v}, \mu)=\inf \{\omega \in \mathbb{R} ; \mathbb{P}(\mathbf{v}, \mu, \omega) \text { is 2-connected }\}
$$

In [6], the authors showed how to reduce the computation of the minimal 2connecting thickness of $(\mathbf{v}, 0)$, where $\mathbf{v}$ is a rational vector, to the one of a smaller vector $\mathbf{v}^{\prime}$ (in terms of norm $\|\cdot\|_{\infty}$ ). Here we extend this reduction to the pairs $(\mathbf{v}, \mu)$ with $\mathbf{v}$ possibly irrational and $\mu \in \mathbb{R}$. 
Theorem 3. Let $\mathbf{v} \in \mathbb{R}_{+}^{3} \backslash\{(0,0,0\}, \mu \in \mathbb{R}$ and $\omega \in \mathbb{R}$. The arithmetical discrete plane $\mathbb{P}(\mathbf{v}, \mu, \omega)$ is 2 -connected if and only if so is $\mathbb{P}\left(\mathbf{v}+v_{1}\left(\mathbf{e}_{\mathbf{2}}+\mathbf{e}_{\mathbf{3}}\right), \mu, \omega+v_{1}\right)$.

In order to prove Theorem 3, we use a technical lemma providing a lower bound of $\Omega_{2}(\mathbf{v}, \mu)$ :

Lemma 3 (A Lower Bound [6]). Let $\mathbf{v} \in \mathbb{R}^{3} \backslash\{(0,0,0\}$ and $\mu \in \mathbb{R}$. We suppose that $\mathbf{v}$ does not have two coordinates equal to zero. Then $\Omega_{2}(\mathbf{v}, \mu) \geq$ $\|\mathbf{v}\|_{\infty}$.

The case where $\mathbf{v}$ has at least one coordinate equal to zero has an explicit answer (see Lemma 4 in Section 5).

Proof (of Theorem 3 ). Let $\mathbf{v}^{\prime}=\mathbf{v}+v_{1}\left(\mathbf{e}_{2}+\mathbf{e}_{3}\right.$ ), let $\omega^{\prime}=\omega+v_{1}$ and let us consider the linear bijection $\Psi_{2}: \mathbb{R}^{3} \longrightarrow \mathbb{R}^{3}$ and its inverse map $\Psi_{2}^{-1}: \mathbb{R}^{3} \longrightarrow \mathbb{R}^{3}$ defined by:

$$
\begin{aligned}
& \Psi_{2}: \mathbb{R}^{3} \longrightarrow \mathbb{R}^{3} \quad \Psi_{2}^{-1}: \mathbb{R}^{3} \longrightarrow \mathbb{R}^{3} \\
& \left(\begin{array}{c}
x_{1} \\
x_{2} \\
x_{3}
\end{array}\right) \longmapsto\left(\begin{array}{c}
x_{1}-x_{2}-x_{3} \\
x_{2} \\
x_{3}
\end{array}\right) \quad \text { and } \quad\left(\begin{array}{c}
x_{1} \\
x_{2} \\
x_{3}
\end{array}\right) \longmapsto\left(\begin{array}{c}
x_{1}+x_{2}+x_{3} \\
x_{2} \\
x_{3}
\end{array}\right)
\end{aligned}
$$

One checks that $\Psi_{2}$ provides a bijection from $\mathbb{P}(\mathbf{v}, \mu, \omega)$ to $\mathbb{P}\left(\mathbf{v}^{\prime}, \mu, \omega\right)$. Moreover, for each $\mathbf{x} \in \mathbb{Z}^{3},\langle\mathbf{v}, \mathbf{x}\rangle=\left\langle\mathbf{v}^{\prime}, \Psi_{2}(\mathbf{x})\right\rangle$.

1. Assume $\mathbb{P}(\mathbf{v}, \mu, \omega)$ is 2-connected. Then, from Lemma 3, it follows that $\omega \geq$ $\|\mathbf{v}\|_{\infty}$. Let us show that $\mathbb{P}\left(\mathbf{v}^{\prime}, \mu, \omega^{\prime}\right)$ is 2-connected too.

Let $\mathbf{x} \in \mathbb{P}\left(\mathbf{v}^{\prime}, \mu, \omega^{\prime}\right)$. Either $\left\langle\mathbf{v}^{\prime}, \mathbf{x}\right\rangle+\mu \in\left[0, \omega\left[\right.\right.$ or $\left\langle\mathbf{v}^{\prime}, \mathbf{x}\right\rangle+\mu \in\left[\omega, \omega^{\prime}[\right.$.

i) If $\left\langle\mathbf{v}^{\prime}, \mathbf{x}\right\rangle+\mu \in\left[0, \omega\left[\right.\right.$, then $\mathbf{x} \in \mathbb{P}\left(\mathbf{v}^{\prime}, \mu, \omega\right)$.

ii) If $\left\langle\mathbf{v}^{\prime}, \mathbf{x}\right\rangle+\mu \in\left[\omega, \omega^{\prime}\right.$, and since $\omega \geq\|\mathbf{v}\|_{\infty} \geq v_{1}$, then $\left\langle\mathbf{v}^{\prime}, \mathbf{x}-\mathbf{e}_{\mathbf{1}}\right\rangle+\mu=$ $\left\langle\mathbf{v}^{\prime}, \mathbf{x}\right\rangle+\mu-v_{1} \in\left[0, \omega\left[\right.\right.$, and $\mathbf{x}-\mathbf{e}_{\mathbf{1}} \in \mathbb{P}\left(\mathbf{v}^{\prime}, \mu, \omega\right)$.

In other words, each $\mathbf{x} \in \mathbb{P}\left(\mathbf{v}^{\prime}, \mu, \omega^{\prime}\right)$ is either in $\mathbb{P}\left(\mathbf{v}^{\prime}, \mu, \omega\right)$ or 2-adjacent to an element of $\mathbb{P}\left(\mathbf{v}^{\prime}, \mu, \omega\right)$. Consequently, it remains to ensure that each pair of elements of $\mathbb{P}\left(\mathbf{v}^{\prime}, \mu, \omega\right)$ is 2-linked in $\mathbb{P}\left(\mathbf{v}^{\prime}, \mu, \omega^{\prime}\right)$. Moreover, since the restriction $\Psi_{2}: \mathbb{P}(\mathbf{v}, \mu, \omega) \longrightarrow \mathbb{P}\left(\mathbf{v}^{\prime}, \mu, \omega\right)$ of $\Psi_{2}$ is one-to-one, then it is sufficient to prove that the images, under $\Psi_{2}$, of two 2-adjacent elements of $\mathbb{P}(\mathbf{v}, \mu, \omega)$ are 2-linked in $\mathbb{P}\left(\mathbf{v}^{\prime}, \mu, \omega^{\prime}\right)$ (see Figure 1).

i) Let $\mathbf{x} \in \mathbb{P}(\mathbf{v}, \mu, \omega)$ such that $\mathbf{x}+\mathbf{e}_{\mathbf{1}} \in \mathbb{P}(\mathbf{v}, \mu, \omega)$. Then $\Psi_{2}\left(\mathbf{x}+\mathbf{e}_{\mathbf{1}}\right)-\Psi_{2}(\mathbf{x})=$ $\mathbf{e}_{\mathbf{1}}$ and the images of $\mathbf{x}$ and $\mathbf{x}+\mathbf{e}_{\mathbf{1}}$ are 2-adajcent in $\mathbb{P}\left(\mathbf{v}^{\prime}, \mu, \omega^{\prime}\right)$.

ii) Let $\mathbf{x} \in \mathbb{P}(\mathbf{v}, \mu, \omega)$ such that $\mathbf{x}+\mathbf{e}_{\mathbf{2}} \in \mathbb{P}(\mathbf{v}, \mu, \omega)$. Hence,

$$
\begin{aligned}
\left\langle\mathbf{v}^{\prime}, \Psi_{2}\left(\mathbf{x}+\mathbf{e}_{1}+\mathbf{e}_{2}\right)\right\rangle+\mu & =\left\langle\mathbf{v}, \mathbf{x}+\mathbf{e}_{\mathbf{1}}+\mathbf{e}_{\mathbf{2}}\right\rangle+\mu \\
& =\left\langle\mathbf{v}, \mathbf{x}+\mathbf{e}_{\mathbf{2}}\right\rangle+\mu+v_{1} \in\left[0, \omega+v_{1}[,\right.
\end{aligned}
$$

since $\mathbf{x}+\mathbf{e}_{\mathbf{2}} \in \mathbb{P}(\mathbf{v}, \mu, \omega)$. Hence $\Psi_{2}\left(\mathbf{x}+\mathbf{e}_{\mathbf{1}}+\mathbf{e}_{\mathbf{2}}\right) \in \mathbb{P}\left(\mathbf{v}^{\prime}, \mu, \omega^{\prime}\right)$. One checks that $\Psi_{2}\left(\mathbf{x}+\mathbf{e}_{\mathbf{1}}+\mathbf{e}_{\mathbf{2}}\right)-\Psi_{2}(\mathbf{x})=\mathbf{e}_{\mathbf{2}}$ and $\Psi_{2}\left(\mathbf{x}+\mathbf{e}_{\mathbf{2}}\right)-\Psi_{2}\left(\mathbf{x}+\mathbf{e}_{\mathbf{1}}+\mathbf{e}_{\mathbf{2}}\right)=-\mathbf{e}_{\mathbf{1}}$. It follows that $\left(\Psi_{2}(\mathbf{x}), \Psi_{2}\left(\mathbf{x}+\mathbf{e}_{1}+\mathbf{e}_{2}\right), \Psi_{2}\left(\mathbf{x}+\mathbf{e}_{\mathbf{2}}\right)\right)$ is a 2-path linking $\Psi_{2}(\mathbf{x})$ to $\Psi_{2}\left(\mathbf{x}+\mathbf{e}_{\mathbf{2}}\right)$ in $\mathbb{P}\left(\mathbf{v}^{\prime}, \mu, \omega^{\prime}\right)$. 


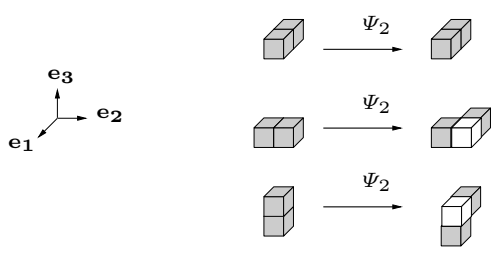

Fig. 1. The action of $\Psi_{2}$ on two 2 -adjacent voxels of $\mathbb{P}(\mathbf{v}, \mu, \omega) .:$ The right grey voxels are the images of the left grey ones, while the right white voxels are elements of $\mathbb{P}\left(\mathbf{v}^{\prime}, \mu, \omega^{\prime}\right)$ which allow us to 2 -link the grey voxels. For ensuring the existence of such white voxels, we remind that the right grey ones belongs to $\mathbb{P}\left(\mathbf{v}^{\prime}, \mu, \omega\right)$ and, for any such voxels $\mathbf{v}$, the (white) voxel $\mathbf{v}+\mathbf{e}_{\mathbf{1}}$ belongs to $\mathbb{P}\left(\mathbf{v}^{\prime}, \mu, \omega^{\prime}\right)$.

iii) A similar reasoning shows that given $\mathbf{x} \in \mathbb{P}(\mathbf{v}, \mu, \omega)$ such that $\mathbf{x}+\mathbf{e}_{\mathbf{3}} \in$ $\mathbb{P}(\mathbf{v}, \mu, \omega), \Psi_{2}(\mathbf{x})$ and $\Psi_{2}\left(\mathbf{x}+\mathbf{e}_{\mathbf{3}}\right)$ are 2-linked in $\mathbb{P}\left(\mathbf{v}^{\prime}, \mu, \omega^{\prime}\right)$.

2. Conversely, suppose $\mathbb{P}\left(\mathbf{v}^{\prime}, \mu, \omega^{\prime}\right)$ is 2-connected. From Lemma 3. one deduces that $\omega^{\prime} \geq\left\|\mathbf{v}^{\prime}\right\|_{\infty}$.

Let $\widetilde{\Psi}_{2}: \mathbb{P}\left(\mathbf{v}^{\prime}, \mu, \omega^{\prime}\right) \longrightarrow \mathbb{P}\left(\mathbf{v}^{\prime}, \mu, \omega\right)$ be the surjective map defined by:

$$
\begin{aligned}
\widetilde{\Psi}_{2}: \mathbb{P}\left(\mathbf{v}^{\prime}, \mu, \omega^{\prime}\right) & \longrightarrow \mathbb{P}(\mathbf{v}, \mu, \omega) \\
\mathbf{v} & \longmapsto \begin{cases}\Psi_{2}^{-1}(\mathbf{x}), & \text { if } \mathbf{x} \in \mathbb{P}\left(\mathbf{v}^{\prime}, \mu, \omega\right) \\
\Psi_{2}^{-1}\left(\mathbf{x}-\mathbf{e}_{\mathbf{1}}\right), & \text { otherwise. }\end{cases}
\end{aligned}
$$

Remind that each $\mathbf{x} \in \mathbb{P}\left(\mathbf{v}^{\prime}, \mu, \omega^{\prime}\right)$ is either in $\mathbb{P}\left(\mathbf{v}^{\prime}, \mu, \omega\right)$ or 2-adjacent to an element of $\mathbb{P}\left(\mathbf{v}^{\prime}, \mu, \omega\right)$. Since $\widetilde{\Psi}_{2}$ is surjective, then it remains to show that the image of two 2-adjacent voxels in $\mathbb{P}\left(\mathbf{v}^{\prime}, \mu, \omega^{\prime}\right)$ are either equal or 2-linked in $\mathbb{P}(\mathbf{v}, \mu, \omega)$ (see Figure 2).

i) Let $\mathbf{x} \in \mathbb{P}\left(\mathbf{v}^{\prime}, \mu, \omega^{\prime}\right)$ such that $\mathbf{x}+\mathbf{e}_{\mathbf{1}} \in \mathbb{P}\left(\mathbf{v}^{\prime}, \mu, \omega^{\prime}\right)$.

- If $\left\langle\mathbf{v}^{\prime}, \mathbf{x}+\mathbf{e}_{\mathbf{1}}\right\rangle+\mu \in\left[0, \omega\left[\right.\right.$, then $\widetilde{\Psi}_{2}\left(\mathbf{x}+\mathbf{e}_{\mathbf{1}}\right)-\widetilde{\Psi}_{2}(\mathbf{x})=\mathbf{e}_{\mathbf{1}}$.

- If $\left\langle\mathbf{v}^{\prime}, \mathbf{x}+\mathbf{e}_{\mathbf{1}}\right\rangle+\mu \in\left[\omega, \omega^{\prime}\left[\right.\right.$, then $\widetilde{\Psi}_{2}\left(\mathbf{x}+\mathbf{e}_{\mathbf{1}}\right)=\widetilde{\Psi}_{2}(\mathbf{x})$.

ii) Let $\mathbf{x} \in \mathbb{P}\left(\mathbf{v}^{\prime}, \mu, \omega^{\prime}\right)$ such that $\mathbf{x}+\mathbf{e}_{\mathbf{2}} \in \mathbb{P}\left(\mathbf{v}^{\prime}, \mu, \omega^{\prime}\right)$.

- If $\left\langle\mathbf{v}^{\prime}, \mathbf{x}+\mathbf{e}_{2}\right\rangle+\mu \in\left[0, \omega\left[\right.\right.$, then $0 \leq\left\langle\mathbf{v}^{\prime}, \mathbf{x}\right\rangle+\mu \leq\left\langle\mathbf{v}^{\prime}, \mathbf{x}\right\rangle+\mu+v_{1}=$ $\left\langle\mathbf{v}^{\prime}, \mathbf{x}+\mathbf{e}_{\mathbf{1}}\right\rangle+\mu \leq\left\langle\mathbf{v}^{\prime}, \mathbf{x}\right\rangle+\mu+v_{1}+v_{2}=\left\langle\mathbf{v}^{\prime}, \mathbf{x}+\mathbf{e}_{\mathbf{2}}\right\rangle+\mu \leq \omega$.

Moreover, $\widetilde{\Psi}_{2}\left(\mathbf{x}+\mathbf{e}_{\mathbf{1}}\right)-\widetilde{\Psi}_{2}(\mathbf{x})=\mathbf{e}_{\mathbf{1}}$ and $\widetilde{\Psi}_{2}\left(\mathbf{x}+\mathbf{e}_{\mathbf{2}}\right)-\widetilde{\Psi}_{2}\left(\mathbf{x}+\mathbf{e}_{\mathbf{1}}\right)=\mathbf{e}_{\mathbf{2}}$ and we have shown that $\left(\widetilde{\Psi}_{2}(\mathbf{x}), \widetilde{\Psi}_{2}\left(\mathbf{x}+\mathbf{e}_{\mathbf{1}}\right), \widetilde{\Psi}_{2}\left(\mathbf{x}+\mathbf{e}_{\mathbf{2}}\right)\right)$ is a 2-path linking $\widetilde{\Psi}_{2}(\mathbf{x})$ to $\widetilde{\Psi}_{2}\left(\mathbf{x}+\mathbf{e}_{\mathbf{2}}\right)$ in $\mathbb{P}(\mathbf{v}, \mu, \omega)$.

- If $\left\langle\mathbf{v}^{\prime}, \mathbf{x}+\mathbf{e}_{\mathbf{2}}\right\rangle+\mu \in\left[\omega, \omega^{\prime}\left[\right.\right.$, then $\widetilde{\Psi}_{2}\left(\mathbf{x}+\mathbf{e}_{\mathbf{2}}\right)=\widetilde{\Psi}_{2}\left(\mathbf{x}+\mathbf{e}_{\mathbf{2}}-\mathbf{e}_{\mathbf{1}}\right)=$ $\widetilde{\Psi}_{2}(\mathbf{x})+\mathbf{e}_{\mathbf{2}}$ and we have shown that $\widetilde{\Psi}_{2}(\mathbf{x})$ and $\widetilde{\Psi}_{2}\left(\mathbf{x}+\mathbf{e}_{\mathbf{2}}\right)$ are 2 -adjacent in $\mathbb{P}(\mathbf{v}, \mu, \omega)$.

Corollary 2. Let $\mathbf{v} \in \mathbb{R}_{+}^{3} \backslash\{(0,0,0)\}$ such that $0 \leq v_{1} \leq v_{2}, v_{3}$, let $\mu \in \mathbb{R}$ and let $\omega \in \mathbb{R}$. The arithmetical discrete plane $\mathbb{P}(\mathbf{v}, \mu, \omega)$ is 2 -connected if and only if so is $\mathbb{P}\left(\mathbf{v}-v_{1}\left(\mathbf{e}_{2}+\mathbf{e}_{3}\right), \mu, \omega-v_{1}\right)$.

In terms of $\Omega_{2}(\mathbf{v}, \mu)$, Theorem 3 and Corollary 2 can be reformulated as follows 


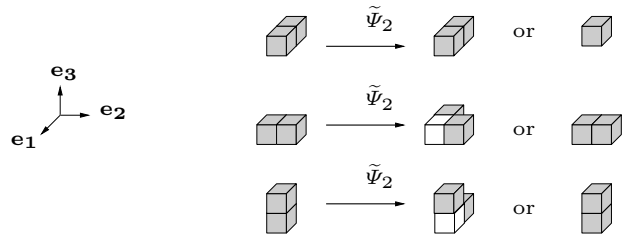

Fig. 2. The action of $\widetilde{\Psi}_{2}$ on two 2-adjacent voxels of $\mathbb{P}\left(\mathbf{v}^{\prime}, \mu, \omega^{\prime}\right)$

Corollary 3. Let $\mathbf{v} \in \mathbb{R}_{+}^{3} \backslash\{(0,0,0)\}$ such that $0 \leq v_{1} \leq v_{2}, v_{3}$ and let $\mu \in \mathbb{R}$. Then

$$
\Omega_{2}(\mathbf{v}, \mu)=\Omega_{2}\left(\mathbf{v}-v_{1}\left(\mathbf{e}_{2}+\mathbf{e}_{3}\right), \mu\right)+v_{1}
$$

\section{Effective Computation of $\Omega_{2}(v, \mu)$}

Let $\mathbf{v} \in \mathbb{R}^{3}$ and $\mu \in \mathbb{R}$. In case of $v_{1}=0$, the reductions given in Theorem 3 . Corollary 2 and Corollary 3 are useless. In fact, in that case we have a direct computation of $\Omega_{2}(\mathbf{v}, \mu)$ since for each $\omega \in \mathbb{R}$,

$$
\mathbb{P}(\mathbf{v}, \mu, \omega)=\bigcup_{k \in \mathbb{Z}}\left(\left\{\mathbf{x} \in \mathbb{Z}^{3} ; x_{1}=0 \text { and } 0 \leq\langle\mathbf{v}, \mathbf{x}\rangle+\mu<\omega\right\}+k \mathbf{e}_{\mathbf{1}}\right) .
$$

Roughly speaking, $\mathbb{P}(\mathbf{v}, \mu, \omega)$ is obtained by translating the arithmetical discrete line $\mathbb{L}\left(\left(v_{2}, v_{3}\right), \mu, \omega\right)$ along the coordinate vector $\mathbf{e}_{\mathbf{1}}$. Hence, in case of $v_{1}=0, \mathbb{P}(\mathbf{v}, \mu, \omega)$ is 2 -connected if, and only if, the arithmetical discrete line $\mathbb{L}\left(\left(v_{2}, v_{3}\right), \mu, \omega\right)$ is 1-connected. From Theorem 2 , it follows:

Lemma 4. Let $\mathbf{v} \in \mathbb{R}^{3} \backslash\{(0,0,0)\}$ and let $\mu \in \mathbb{R}$. If $v_{1}=0$, then $\Omega_{2}(\mathbf{v}, \mu)=$ $\Omega_{1}\left(\left(v_{2}, v_{3}\right), \mu\right)$. More precisely,

$$
\begin{array}{ll}
\Omega_{2}(\mathbf{v}, \mu)=\mu-v_{3}\left\lfloor\frac{\mu}{v_{3}}\right\rfloor, & \text { if } v_{2}=0 \text { and } v_{3} \neq 0, \\
\Omega_{2}(\mathbf{v}, \mu)=\mu-v_{2}\left\lfloor\frac{\mu}{v_{2}}\right\rfloor, & \text { if } v_{2} \neq 0 \text { and } v_{3}=0, \\
\Omega_{2}(\mathbf{v}, \mu)=\|\mathbf{v}\|_{1}-v_{2} \wedge v_{3}, & \text { if } v_{2} \neq 0 \text { and } v_{3} \neq 0 .
\end{array}
$$

REMARK - Of course, the other cases $\left(v_{2}=0\right.$ and $\left.v_{3}=0\right)$ are similar.

Let us now treat the general case. For clarity issues, and with no loss of generality, we reduce the computation of $\Omega_{2}(\mathbf{v}, \mu)$ to the vectors $\mathbf{v} \in$ $\left\{\mathbf{x} \in \mathbb{R}_{+}^{3}, 0 \leq x_{1} \leq x_{2} \leq x_{3}\right\}^{\star}=\left\{\mathbf{x} \in \mathbb{R}_{+}^{3}, 0 \leq x_{1} \leq x_{2} \leq x_{3}\right\} \backslash\{(0,0,0)\}$. In fact, up to an isometry of the unit cube $[-0.5,0.5]^{3}$, for each vector $\mathbf{v} \in \mathbb{R}^{3}$ and each $\mu \in \mathbb{R}$, there exists a vector $\mathbf{v}^{\prime} \in \mathbb{R}^{3}$ such that $0 \leq v_{1}^{\prime} \leq v_{2}^{\prime} \leq v_{3}^{\prime}$ and $\Omega_{2}(\mathbf{v}, \mu)=\Omega_{2}\left(\mathbf{v}^{\prime}, \mu\right)$. 
Notation - Let $\Delta: \mathbb{R}^{3} \longmapsto \mathbb{R}_{+}^{3}$ the map defined by:

$$
\begin{aligned}
& \Delta:\left\{\mathbf{x} \in \mathbb{R}_{+}^{3}, 0 \leq x_{1} \leq x_{2} \leq x_{3}\right\} \longmapsto\left\{\begin{array}{l}
\left.\mathbf{x} \in \mathbb{R}_{+}^{3}, 0 \leq x_{1} \leq x_{2} \leq x_{3}\right\} \\
\left(\begin{array}{c}
x_{1} \\
x_{2}-x_{1} \\
x_{3}-x_{1}
\end{array}\right) \text { if } x_{1} \leq x_{2}-x_{1} \leq x_{3}-x_{1}, \\
x_{2} \\
x_{3}
\end{array}\right) \\
& \mapsto\left(\begin{array}{c}
x_{2}-x_{1} \\
x_{1} \\
x_{3}-x_{1}
\end{array}\right) \text { if } x_{2}-x_{1} \leq x_{1} \leq x_{3}-x_{1}, \\
&\left(\begin{array}{c}
x_{2}-x_{1} \\
x_{3}-x_{1} \\
x_{1}
\end{array}\right) \text { otherwise. }
\end{aligned}
$$

Roughly speaking, given a normal vector $\mathbf{v} \in\left\{\mathbf{x} \in \mathbb{R}_{+}^{3}, 0 \leq x_{1} \leq x_{2} \leq x_{3}\right\}$, the operator $\Delta$ subtracts the lowest coordinate of $\mathbf{v}$ to the other ones and reorder the output vector. This operator is known as the fully subtractive algorithm, a two-dimensional continued fraction algorithm. For more informations, one can refer to 8 .

Notation - Let $\mathbf{v} \in\left\{\mathbf{x} \in \mathbb{R}_{+}^{3}, 0 \leq x_{1} \leq x_{2} \leq x_{3}\right\}$ and let $n \in \mathbb{N}$. We set $\mathbf{v}^{(n)}=\Delta^{n}(\mathbf{v}) \in\left\{\mathbf{x} \in \mathbb{R}_{+}^{3}, 0 \leq x_{1} \leq x_{2} \leq x_{3}\right\}$.

Let $\mathbf{v} \in\left\{\mathbf{x} \in \mathbb{R}_{+}^{3}, 0 \leq x_{1} \leq x_{2} \leq x_{3}\right\}, \mu \in \mathbb{R}$ and $n \in \mathbb{N}$. From Corollary 3 , it follows

$$
\|\mathbf{v}\|_{1}-2 \Omega_{2}(\mathbf{v}, \mu)=\left\|\Delta^{n}(\mathbf{v})\right\|_{1}-2 \Omega_{2}\left(\Delta^{n}(\mathbf{v}), \mu\right) .
$$

Given $\mathbf{v} \in\left\{\mathbf{x} \in \mathbb{R}_{+}^{3}, 0 \leq x_{1} \leq x_{2} \leq x_{3}\right\}$, the sequences $\left(v_{1}^{(n)}\right)_{n \in \mathbb{N}},\left(v_{2}^{(n)}\right)_{n \in \mathbb{N}}$ and $\left(v_{3}^{(n)}\right)_{n \in \mathbb{N}}$ are non-increasing and minored by 0 . Hence they are convergent. Let $\mathbf{v}^{\prime}=\lim _{n \rightarrow \infty} \Delta^{n}(\mathbf{v})$, then $\mathbf{v}^{\prime}=\Delta\left(\mathbf{v}^{\prime}\right)$ implies $v_{1}^{\prime}=0$ and one deduces:

Theorem 4. Let $\mathbf{v} \in\left\{\mathbf{x} \in \mathbb{R}_{+}^{3}, 0 \leq x_{1} \leq x_{2} \leq x_{3}\right\}^{\star}$, let $\mu \in \mathbb{R}$ and let $\mathbf{v}^{\prime}=$ $\lim _{n \rightarrow \infty} \Delta^{n}(\mathbf{v})$. Then,

$$
\Omega_{2}(\mathbf{v}, \mu)=\frac{\|\mathbf{v}\|_{1}-\left\|\mathbf{v}^{\prime}\right\|_{1}+2 \Omega_{2}\left(\mathbf{v}^{\prime}, \mu\right)}{2} .
$$

REMARK - Theorem 4 provides an explicit process to compute $\Omega_{2}(\mathbf{v}, \mu)$ whatever $\mathbf{v} \in \mathbb{R}^{3}$ and $\mu$. Indeed, since $v_{1}^{\prime}=0$, thanks to Lemma 4 , we can explicitly compute $\Omega_{2}\left(\mathbf{v}^{\prime}, \mu\right)$.

Given $\mathbf{v} \in\left\{\mathbf{x} \in \mathbb{R}_{+}^{3}, 0 \leq x_{1} \leq x_{2} \leq x_{3}\right\}$, two cases occur:

1. there exists $n_{0} \in \mathbb{N}$ such that $v_{1}^{\left(n_{0}\right)}+v_{2}^{\left(n_{0}\right)}<v_{3}^{\left(n_{0}\right)}$,

2. for all $n_{0} \in \mathbb{N}$, there exists $n \geq n_{0}$ such that $v_{1}^{(n)}+v_{2}^{(n)} \geq v_{3}^{(n)}$.

From Theorem 4 , one deduces the following Corollaries. 
Corollary 4 (The first case). Let $\mathbf{v} \in\left\{\mathbf{x} \in \mathbb{R}_{+}^{3}, 0 \leq x_{1} \leq x_{2} \leq x_{3}\right\} \backslash$ $\{(0,0,0)\}$, let $\mu \in \mathbb{R}$ and let $n_{0} \in \mathbb{N}$ such that $v_{1}^{\left(n_{0}\right)}+v_{2}^{\left(n_{0}\right)}<v_{3}^{\left(n_{0}\right)}$.

i) If $v_{1}^{\left(n_{0}\right)} \wedge v_{2}^{\left(n_{0}\right)}=0$, then:

$$
\Omega_{2}(\mathbf{v}, \mu)=\frac{\|\mathbf{v}\|_{1}-\gamma}{2}+\left(\mu-\gamma\left\lfloor\frac{\mu}{\gamma}\right\rfloor\right) \text {, with } \gamma=v_{3}^{\left(n_{0}\right)}-\left(v_{1}^{\left(n_{0}\right)}+v_{2}^{\left(n_{0}\right)}\right) \text {. }
$$

ii) If $v_{1}^{\left(n_{0}\right)} \wedge v_{2}^{\left(n_{0}\right)} \neq 0$, then:

$$
\Omega_{2}(\mathbf{v}, \mu)=\frac{\|\mathbf{v}\|_{1}-\left(v_{1}^{\left(n_{0}\right)}+v_{2}^{\left(n_{0}\right)}\right)+v_{3}^{\left(n_{0}\right)}+2\left(v_{1}^{\left(n_{0}\right)} \wedge v_{2}^{\left(n_{0}\right)}-v_{1}^{\left(n_{0}\right)} \wedge v_{2}^{\left(n_{0}\right)} \wedge v_{3}^{\left(n_{0}\right)}\right)}{2} .
$$

REMARK - The main advantage of Corollary 4 is to provide a possibly finite process to compute $\Omega_{2}(\mathbf{v}, \mu)$ for a totally irrational vector $\mathbf{v}$, that is, satisfying $\operatorname{dim}_{\mathbb{Q}}\left\{v_{1}, v_{2}, v_{3}\right\}=3$.

Corollary 5 (The Second Case). Let $\mathbf{v} \in\left\{\mathbf{x} \in \mathbb{R}_{+}^{3}, 0 \leq x_{1} \leq x_{2} \leq x_{3}\right\}$ and assume:

$$
\forall n_{0} \in \mathbb{N}, \exists n \geq n_{0}, v_{1}^{(n)}+v_{2}^{(n)}>v_{3}^{(n)} .
$$

Then

$$
\Omega_{2}(\mathbf{v}, \mu)=\frac{v_{1}+v_{2}+v_{3}}{2}
$$

\section{Conclusion}

In the present paper we investigated, given $\mathbf{v} \in \mathbb{R}^{3}$ and $\mu \in \mathbb{R}$, the computation of $\inf \{\omega \in \mathbb{R}, \mathbb{P}(\mathbf{v}, \mu, \omega)$ is 2 -connected $\}$. The main improvement we made, compare to [6] is that we do not treat the rational case and the irrational case separately but in a common way and in a unique expression, thanks to the introduction of a notation inherited from the (multidimensional) continued fractions theory.

Moreover, we have shown the role of the translation parameter $\mu$ in the computation of $\Omega_{2}(\mathbf{v}, \mu)$. This result was already known for the rational arithmetical discrete planes but not for the irrational ones.

Due to the limitation of space, we do not have included the investigation of the 0 -connectedness and the 1-connectedness of arithmetical discrete planes. In fact, this studies are similar to the one we did in the present paper. For explicit formulas see [6]. Reformulating in terms of possibly irrational arithmetical discrete planes is obvious.

In a further work, it will be interesting to extend all the present results to arithmetical discrete hyperplanes in any dimension. Another interesting way to investigate is the behavior of the class of arithmetical discrete (hyper)planes under the action of other multidimensional continued fractions algorithms. 


\section{References}

1. Reveillès, J.P.: Géométrie discrète, calcul en nombres entiers et algorithmique. Thèse d'état, Université Louis Pasteur, Strasbourg (1991)

2. Andres, E., Acharya, R., Sibata, C.: Discrete analytical hyperplanes. CVGIP: Graphical Model and Image Processing 59(5), 302-309 (1997)

3. Gérard, Y.: Periodic graphs and connectivity of the rational digital hyperplanes. Theor. Comput. Sci. 283(1), 171-182 (2002)

4. Brimkov, V., Barneva, R.: Connectivity of discrete planes. Theor. Comput. Sci. 319(1-3), 203-227 (2004)

5. Jamet, D., Toutant, J.L.: On the connectedness of rational arithmetic discrete hyperplanes. In: Kuba, A., Nyúl, L.G., Palágyi, K. (eds.) DGCI 2006. LNCS, vol. 4245, pp. 223-234. Springer, Heidelberg (2006)

6. Jamet, D., Toutant, J.L.: Minimal arithmetic thickness connecting discrete planes. Discrete Applied Mathematics 157(3), 500-509 (2009)

7. Berthé, V., Fiorio, C., Jamet, D., Philippe, F.: On some applications of generalized functionality for arithmetic discrete planes. Image Vision Comput 25(10), 1671-1684 (2007)

8. Schweiger, F.: Multidimensional continued fractions. Oxford Science Publications. Oxford University Press, Oxford (2000) 